Originalveröffentlichung in: D. Henke et al. (Hg.), Der „ganze Mensch“. Perspektiven

lebensgeschichtlicher Individualität, Festschrift für Dietrich Rößler. Arbeiten zur

Praktischen Theologie 10, Berlin, 1997, S. 13-18

WolfGang Röllig (Tübingen)

\title{
Altorientalische Schiffsmetaphorik
}

In Hirbet Bēt Layy, etwa $8 \mathrm{~km}$ östlich von Lachisch gelegen, wurde 196r eine Grabhöhle entdeckt, die neben einer Anzahl althebräischer Inschriften ${ }^{1}$ des 7. Jh.v.Chr. auch grob eingeritzte Zeichnungen von Menschen, einem Militärcamp(?) und von Schiffen an den Wänden des Vorraums des doppelten Kammergrabes erkennen läß ${ }^{2}$. Die Texte enthalten eine zweizeilige Prädikation Jahwes, mehrere knappe Fluchformeln und - ganz in der Nähe der Schiffsdarstellungen - zwei Anrufungen mit Bittformeln: „Schreite ein, Jah, gnädiger Gott! / Erkläre straffrei, Jah, Jahwe!“ und „Errette, [J]ahwe!“ Es ist zwar keinesfalls sicher, daß zwischen den Darstellungen und den Inschriften ein Zusammenhang besteht, denn die Texte sind nicht so angebracht, daß man den Eindruck hat, sie würden durch die Zeichnungen illustriert. Dennoch wird man kaum ein zufälliges Ensemble vermuten und nach Deutungen der möglichen Zusammenhänge suchen. Dies auch deshalb, weil man sich fragen muß, was Schiffsdarstellungen im südlichen Palästina, am inneren Rande der Schefela, rund $40 \mathrm{~km}$ vom Ufer des Mittelmeeres entfernt, eigentlich besagen sollen ${ }^{3}$. Daß man gerade dort und noch dazu in einem Grab eventuell die Rettung aus Seenot erflehte, ist nicht

Zuletzt behandelt in Johannes Renz / Wolfgang Röllig: Handbuch der althebräischen Epigraphik, Darmstadt 1995, Bd. I, 242-251: BLay 7,I-7. Dort auch die frühere Literatur zu den Fundumständen.

2 Zu den Darstellungen s. vor allem Joseph Naveh: Israel Exploration Journal 13, Jerusalem 1963, 74-92; Siegfried Mittmann: Acta Academica. Journal for Human Sciences Research, Bloemfontein, 21 (1989), 15-38.

3 Siegfried Mittmann, der die Texte und Darstellungen in den historischen Zusammenhang der Belagerung Jerusalems durch Sanherib im Jahr 70 or v.Chr. stellen möchte, versucht die Erklärung: "The depiction of ships may indicate that the Assyrians forced some Phoenician cities under their control to assist them with their fleet in the Assyrian campaign." In dem Feldzugsbericht Sanheribs (Daniel D. Luckenbill: Annals of Sennacherib, Chicago 1924, I68ff. II 37- III 49, deutsche Übersetzung z.B. bei Kurt Galling: Textbuch zur Geschichte Israels, Tübingen $\left.{ }^{2} 1968,67-69\right)$ wird davon allerdings nichts erwähnt. 
überaus wahrscheinlich. Deshalb ist es wohl erlaubt, auch über die Grenzen Palästinas herauszuschauen nach Darstellungen oder Texten, in denen Schiffsmetaphorik und Errettungswunsch miteinander verknüpft sind, und nach deren Bedeutung zu fragen.

Einer der nicht sehr zahlreichen Texte assyrischer Provenienz, der eine individuelle Klage enthält, ist ein ergreifendes Gedicht ${ }^{4}$, das in der Form eines Dialoges beginnt:

"WWarum bist du wie ein Schiff inmitten des Stromes festgehalten,

sind deine Rahen zerbrochen, abgeschnitten deine Taue?

Bedeckt ist dein Antlitz, den Fluß der ,Inneren Stadt t' überschreitest du.» "Wie sollte ich nicht festgehalten sein, sollten meine Taue nicht gekappt sein?

Am Tage, an dem ich Frucht trug - wie freute ich mich!

Froh war ich, froh war mein Gatte.

(Aber) am Tage meiner Niederkunft wurde mein Antlitz umwölkt,

am Tage, als ich gebären sollte, wurden meine Augen verschleiert.

Mit geöffnet (erhobenen) Händen betete ich zu Bëlet-ili:

,Du bist auch eine Mutter, die gebar, rette mein Leben!'»"

Die Göttin jedoch, die die Schöpfergöttin selbst ist, verschleiert ihr Antlitz vor der Kreißenden und „der Tod schlich sich in mein Schlafgemach, trieb mich aus meinem Haus, riß mich von der Seite meines Gatten“. Die Geburt, so freudig erwartet, endet tragisch; die Wöchnerin stirbt im Kindbett, überschreitet den Fluß, - wahrscheinlich indirekt eine Anspielung auf den akkadischen Unterweltsfluß Hubur ${ }^{6}$. Das war im Mesopotamien der baby-

4 K. 890 (Tafelsignatur der Kouyunjik Collection des British Museum, London), publiziert von S.A. Strong: Beiträge zur Assyriologie 2, 1894, 634, zuletzt behandelt von Erica Reiner, in: Wolfgang Röllig (Hg.): Altorientalische Literaturen. Neues Handbuch der Literaturwissenschaft I, Wiesbaden 1978, 186f.; dies.: Your Thwarts in Pieces - Your Mooring Rope Cut. Poetry from Babylonia and Assyria, Michigan 1985, 85-93 (Photo auf dem cover); Rainer Albertz: Persönliche Frömmigkeit und offizielle Religion. Religionsinterner Pluralismus in Israel und Babylon, Stuttgart 1978, 54; Karl Hecker in: Otto Kaiser (Hg.): Texte aus der Umwelt des Alten Testaments, Band 2, Gütersloh 1986/91, 780f.; Benjamin Read Foster: Before the Muses II, Ann Arbor 1993, 905.

s Mit libbi äli „Inneres der Stadt" wird die assyrische Hauptstadt Assur häufig bezeichnet, die am „Fluß“, dem Tigris, gelegen ist. Hier ist offenbar gemeint, daß die Klagende sich aus der menschlichen Gemeinschaft in der Stadt entfernt, hinaus in die Steppe, in das Reich der Dämonen und des Todes geht.

6 Vgl. Wolfgang Röllig: Reallexikon der Assyriologie Band 4, 1972/75, 478f. - Zum auch ikonographischen Motiv des Bootes bei der Fahrt in die Unterwelt s. schon J.J.A. van Dijk: Fremdsprachige Beschwörungstexte in der südmesopotamischen literarischen Überlieferung, in: Hans-Jörg Nissen - Johannes Renger ( $\mathrm{Hg}$.): Mesopotamien und seine Nachbarn. Berliner Beiträge zum Vorderen Orient I/I, Berlin 1982, 104. 
lonisch-assyrischen Zeit ein sicher häufiges Ereignis ${ }^{7}$. Deshalb überrascht es nicht, daß man versuchte, sich so gut wie möglich dagegen zu schützen. Das war u.a. dadurch möglich, daß man während der Schwangerschaft Amulette gegen Lamaštu, die Dämonin des Kindbettfiebers, trug, daß man bei der Geburt Beschwörungen rezitierte wie z.B. diejenige, in der der Mythos vom Mondgott ${ }^{8}$ und der Kuh erzählt wird ${ }^{9}$. Er handelt ja von den höchst schmerzhaften Geburtswehen der Geliebten des Gottes, die schließlich - mit Lebenswasser besprengt und mit Öl gesalbt - leicht wie eine Gazelle niederkommt. Eine solche Geburt war es dann, die man der Wöchnerin wünschte - keine, „in der die Gebärende mit Todesstaub bedeckt" ist ${ }^{\text {IO }}$.

Das Bild vom Schiff, beladen mit der teuren Last des Kindes während der Schwangerschaft, findet sich aber noch an anderer Stelle. Auf einer großen Tafel mit Rezepten und Beschwörungen zur Erleichterung der Geburt, die in Assur gefunden wurde, heißt es u.a.:

„Auf dem ... Weg möge das Schiff heil ankommen auf dem Weg seines ... möge das Lastschiff recht vorankommen.

Möge ihr festes Tau gelöst werden und ihr verschlossenes Tor ${ }^{\text {t1 }}$ geöffnet werden.

7 Es wurde - in Anspielung auf altorientalische Sitten - in höchst eindrucksvoller Weise auch in der Szene von Rahels Tod geschildert von Thomas Mann in "Joseph und seine Brüder“ „Die Geschichten Jaakobs. Siebentes Hauptstück: Benoni“. In einer späteren Tagebucheintragung bekennt der Dichter, daß er beim Wiederlesen der Szene in Tränen ausgebrochen sei.

8 Es ist natürlich kein Zufall, daß hier gerade der Mondgott herangezogen wird. Die Leibesfrucht (inbu), von der auch in der Elegie die Rede ist, ist assoziativ mit dem Mond zu verbinden, von dem es u.a. heißt: „Frucht (inbu), die aus sich selbst wächst, die an Umfang zunimmt, die lieblich anzusehen ist, an deren Fülle man sich nicht sättigen kann“ IV R (= H. Rawlinson u.a., The Cuneiform Inscriptions of Western Asia, London) 9,22f., vgl. auch den Namen der hemerologischen Serie „Frucht (inbu), Herr des Neumondes" in IV R 33 IV 13 und R.F. Harper: Assyrian and Bablyonian Letters, London / Chicago, 362 =: Simo Parpola u.a., State Archives of Assyria, Helsinki, 10,22I Rs. 7 usw. und s. The Assyrian Dictionary of the University of Chicago, Glückstadt, I/J $1960,146$.

9 Dazu zuletzt W. Faber: „Die Kuh des Sin»: Ritual für schwierige Geburt“, in: Otto Kaiser (Hg.): Texte aus der Umwelt des Alten Testaments Bd.2, Gütersloh 1986/91, 274277; N. Veldhuis: The Cow of Sin, Groningen r991.

to So nach einer mittelassyrischen Beschwörung veröffentlicht von Wilfred G. Lambert: Iraq 31, 1969, 3if., Z.37.

"Benjamin Read Foster (vgl. die folgende Fußnote) nimmt auch hier eine Fortsetzung der Schiffsmetaphorik an und übersetzt "her battened amidships be opened“. Aber das Bild vom verschlossenen Tor für die schwere Geburt findet sich auch in der eben zitierten Beschwörung: Iraq 31, 3I f., Z.35f: mahis sikküru - saniq bäbu „vorgelegt ist der Riegel, verschlossen die Tür!“. 
Das Tau des Schiffes am Kai des Heils,

Das Tau des Lastschiffes am Kai des Lebens!

Mögen die Glieder gelöst werden, die Sehnen erschlaffen,

möge der versiegelte (Mutterleib) gelöst werden, das Kind herauskommen!

Möge das fremde Gebilde, das menschliche Geschöpf

schleunigst herauskommen und das Licht der Sonne erblicken! ${ }^{\text {it2 }}$

Der Text ist weitgehend aus sich heraus verständlich: Die Schwangere befindet sich in Geburtswehen, aber die Leibesfrucht will nicht abgehen, der Gebärvorgang stoppt. Wie ein Schiff am Kai vertäut ist, sich nicht vorwärts bewegen kann, so ist das Kind im Inneren der Mutter festgebunden - oder, wie eine andere Stelle im gleichen Text besagt:

„Am Kai des Todes ist das Schiff zurückgehalten, am Kai der Not ist das Lastschiff zurückgehalten.

[Deshalb flehe ich] zu Ea, dem Herrn der Beschwörung:

[Vom Kai des Todes] möge er das Schiff lösen,

[vom Kai der Not möge] er das Lastschiff los machen!" ${ }^{(13}$

Nur durch eine Beschwörung ist es also möglich, das "Schiff" vom Kai ablegen zu lassen, die Geburt zu befördern. Dann wird aus dem Kai des

12 Franz Köcher: Die babylonisch-assyrische Medizin in Texten und Untersuchungen, Berlin, 248 II 46-54, s. dazu schon E. Ebeling: Archiv für die Geschichte der Medizin 14 (1923) 68f.; Benjamin Read Foster: Before the Muses II, Ann Arbor 1993, 893.

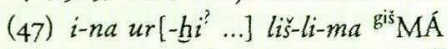

(48) $i-n a$ ur $\left[-h i^{?} \ldots \zeta^{\prime}\right]$ u liš-te-še-ra ${ }^{\text {giš MÁ.GUR }}$

(49) dan-nu lip-pa-tir mar-kas-sa

(so) ì ed-lu lip-pe-ti KÁ-šá

(sI) DUR ša giš MA a-na kar šul-me

(52) DUR sa ${ }^{\text {gis }}{ }^{\text {MÁ.GUR }} \mathrm{GU}_{8}$ a-na kar TI.LA

(53) meš-re-e-tu lip-te-ți-ra li-ir-mu-ú $\mathrm{SA}^{\mathrm{mcs}}$

(54) ka-an-ga-tu lip-taś-si-ra li-sa-a nab-ni-tu

(55) GIR.PAD.DU $a-h i-t u_{4} b i-n u-u t a-m e-l[u-] t i$

(56) ár-his li-ta-sa-am-ma li-ta-mar ZALAG ${ }^{\mathrm{d}} \mathrm{UTU}$-ši.

13 F. Köcher: Die babylonisch-assyrische Medizin in Texten und Untersuchungen, Berlin, 248 III $58-62$ :

(58) ina kar mu-ti ka-lat gišMÁ

(59) i[na k]ar dan-na-ti ka-lat gišMÁ.GUR

(60) [ ] ] ana ${ }^{\mathrm{d}} 40 \mathrm{EN}$ EN

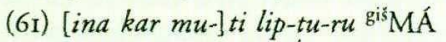

(62) [ina kar dan-na-ti li-] ram-ma-a ${ }^{\text {gis } M A ́ . G U R}$. 
Todes ein solcher des Heils, des Lebens, denn Mutter und Kind können am Leben bleiben. Die Glieder müssen dafür gelockert werden, die Sehnen entspannt, so daß das "Tor" von seinem gesiegelten Torverschluß befreit wird, das Kind den Muttermund verläßt. Geschieht das nicht, bleibt das Schiff inmitten des Stromes stecken, folgt der Tod.

Das Todesschicksal ist es wohl auch, das die Aussage einer zweisprachigen Tammuzklage bestimmt, die in dieser Form erst aus recht junger Zeit stammt, d.h. in neuassyrischer Zeit niedergeschrieben wurde. In ihrem ersten Teil ist von dem Verfallensein des jugendlichen Gottes an die „Brust der Erde", d.h. die Unterwelt die Rede. Im zweiten, litaneiartig wiederholten Teil ist die Aussage dahingehend modifiziert, daß es heißt:

„Seine Kleinen liegen in einem gesunkenen Boot, seine Großen liegen in der überschwemmten Ernte ${ }^{\text {“14. }}$.

Auch hier kann das Bild vom versunkenen Schiff schwerlich etwas anderes meinen, als den Mutterleib mit dem in ihm gestorbenen Kind, das nun ebenso wie Dumuzi in der Unterwelt weilt. Die Erwachsenen aber sind dahingerafft wie die von einer Flut oder vom Gewittersturm niedergewalzte Getreideernte.

Kehren wir zu unserem Ausgangspunkt zurück, so halte ich es für möglich, wenn auch nicht im strengen Sinne für bewiesen, daß die beiden Schiffsdarstellungen in dem Grab von Hirbet Bēt Layy nebst den beiden Anrufungen Jahwes zur Errettung aus der Not sich auch auf eine Situation beziehen, in der eine Frau in tödlichen Geburtswehen ihren Gott anrief. Die Figur eines Beters oder einer Beterin mit erhobenen Händen, die sich unweit der Schiffsdarstellungen ebenfalls an der Südwand des Grabes eingeritzt fand, könnte diesen Zusammenhang noch unterstreichen. Damit wären diese Darstellungen aber aus jedem historischen Kontext herausgenommen und die flehenden Bitten um Errettung hätten einen ganz individuellen Chrarakter.

Die Schiffsmetaphorik erlischt natürlich nicht mit den Kulturen des Alten Orients. Ein Zeugnis, das auch noch den Zusammenhang mit der Geburt bewahrt hat, aber doch wohl von der altorientalischen Tradition

14 IV R 30 Nr.2 Rs.Iof. und Dupl. SBH 67: Nr.37,2If.

si-ih-hi-ru-tu-šú ina eleppi tebition nilu

rab-bu-tu-šú ina ebüri šal-lu-ma nìlu

sum.: TUR.TUR.bi giš.má sù.sù i/in.ná

gal.gal.bi EBUR sù.sù/su.su i/in.ná. - Zu šallu „überschwemmt“ s. The Assyrian Dictionary of the University of Chikago, Glückstadt, $\check{S}_{1} 253 \mathrm{~b}$. 
völlig unabhängig ist, findet sich noch bei dem spätantiken Schriftsteller Fabius Planciades Fulgentius (5.Jh.n.Chr.) ${ }^{15}$, der im ersten Buch seiner "Expositio Virgilianae“, einer allegorischen und etymologischen Deutung der Aeneis, den Schiffbruch des Aeneas als die Geburt des Menschen deutet $^{16}$. Hier ist es der Sturm und die daraus resultierende Turbulenz, was den Vergleich mit der gefährlichen Geburt anregt.

Schwangerschaft und Schiffssymbolik verbindet aber auch in einer sehr tief gefühlten Weise der Text des Adventsliedes von Johannes Tauler, in dem es heißt:

„Es kommt ein Schiff geladen, bis an sein höchsten Bord, trägt Gottes Sohn voll Gnaden, des Vaters ewigs Wort" usw.

Das Lebensschiff, der Mutterleib, der in der altorientalischen Metaphorik des vertäuten und gelösten Schiffes, des beim Scheitern an Tauen und Rahen gebrochenen Bootes als Bild vorweggenommen ist, erhält hier seinen letzten Sinn: Es ist das Gefäß, das das göttliche Wort, den Gottessohn selbst aufgenommen hat und zur Welt bringen wird.

15 Ich verdanke den Hinweis auf diese Stelle Herrn Kollegen Günter Wille, Tübingen.

16 Nach Martin Schanz / Carl Hosius / Gustav Krüger: Geschichte der römischen Literatur, in: Walter Otto, Handbuch der Altertumswissenschaft, 8.Abt., Hd.4/2, München 1922, 198f., $\$ 1096$. 